\title{
Essential residues for the enzyme activity of ATP-dependent MurE ligase from Mycobacterium tuberculosis
}

\author{
Chandrakala Basavannacharya, Paul R. Moody, Tulika Munshi, Nora Cronin, Nicholas H. Keep, \\ Sanjib Bhakta ${ }^{\bowtie}$
}

Institute for Structural and Molecular Biology, Department of Biological Sciences, Birkbeck, University of London, London WC1E 7HX, UK

\ Correspondence: s.bhakta@bbk.ac.uk

Received October 19, 2010 Accepted October 31, 2010

\section{ABSTRACT}

The emergence of total drug-resistant tuberculosis (TDRTB) has made the discovery of new therapies for tuberculosis urgent. The cytoplasmic enzymes of peptidoglycan biosynthesis have generated renewed interest as attractive targets for the development of new antimycobacterials. One of the cytoplasmic enzymes, uridine diphosphate (UDP)-MurNAc-tripeptide ligase (MurE), catalyses the addition of meso-diaminopimelic acid ( $m$-DAP) into peptidoglycan in Mycobacterium tuberculosis coupled to the hydrolysis of ATP. Mutants of $M$. tuberculosis MurE were generated by replacing K157, E220, D392, R451 with alanine and N449 with aspartate, and truncating the first 24 amino acid residues at the $\mathrm{N}$ terminus of the enzyme. Analysis of the specific activity of these proteins suggested that apart from the $24 \mathrm{~N}$ terminal residues, the other mutated residues are essential for catalysis. Variations in $K_{m}$ values for one or more substrates were observed for all mutants, except the $\mathrm{N}$-terminal truncation mutant, indicating that these residues are involved in binding substrates and form part of the active site structure. These mutant proteins were also tested for their specificity for a wide range of substrates. Interestingly, the mutations K157A, E220A and D392A showed hydrolysis of ATP uncoupled from catalysis. The ATP hydrolysis rate was enhanced by at least partial occupation of the uridine nucleotide dipeptide binding site. This study provides an insight into the residues essential for the catalytic activity and substrate binding of the ATP-dependent MurE ligase. Since ATPdependent MurE ligase is a novel drug target, the understanding of its function may lead to development of novel inhibitors against resistant forms of M. tuberculosis.

KEYWORDS peptidoglycan, MurE ligase, site-directed mutagenesis, $m$-DAP

\section{INTRODUCTION}

Tuberculosis is an increasing global health problem. The emergence of totally drug-resistant (TDR) strains of Mycobacterium tuberculosis poses a serious challenge, requiring the discovery of new anti-TB drugs against these strains with potential novel mechanisms of action (Velayati et al., 2009). Peptidoglycan, part of the cell envelope of $M$. tuberculosis, plays a major role in preserving the structural integrity of the cell by withstanding the high internal osmotic pressure. It is a giant macromolecule located on the outside of the cytoplasmic membrane in most of eubacteria (Schleifer and Kandler, 1972). In M. tuberculosis, the peptidoglycan consists of linear glycan chains with alternating units of $N$-acetyl- $\alpha-D$ glucosamine (GlcNAc) and $\mathrm{N}$-acetyl/N-glycolyl muramic acid (MurNAc/MurNGI) substituted with a peptide side-chain that may be cross-linked to peptide of other glycan strands (Mahapatra et al., 2005a). Due to their absence from animal cells, peptidoglycan biosynthesis enzymes are attractive targets for antimicrobials.

Peptidoglycan biosynthesis is a complex process involving 
three major steps. In the first step, MurA and subsequently MurB convert UDP-GIcNAc to UDP-MurNAc. The next step is catalyzed by four ATP-dependent Mur ligases, MurC, MurD, MurE and MurF which catalyze the addition of L-ala, D-glu, $m$ DAP/L-lys and D-ala-D-ala sequentially with the hydrolysis of ATP. These ligases share a common reaction mechanism (Barreteau et al., 2008).

From a multiple sequence alignment of MurC, MurD, MurE and MurF ligases from E. coli, conserved residues were identified, which suggested that these genes evolved from a common ancestral gene (lkeda et al., 1990). Ten invariant amino acids, including 3 residues in the ATP binding consensus sequence GxxGKT/G were recognized by comparing amino acid sequences of 20 Mur synthetases including MurC, D, E, F, Mpl, folylpoly-Y-L-glutamate synthetase (FPGS or FolC) from several bacteria, the C-terminal domain of cyanophycin synthetase (CphA) from Synechocystis sp. and the poly- $\gamma$-glutamate synthetase (CapB) from Bacillus anthracis (Bouhss et al., 1997; Bouhss et al., 1999). To evaluate the roles of these conserved residues in catalysis and binding of the substrates in the Mur superfamily, site-directed mutagenesis of these invariants were studied using MurC and MurD from E. coli (Bouhss et al., 1997; Bouhss et al., 1999). This study was facilitated by the availability of 3-D structural information for MurD in the presence of ligands (Bertrand et al., 1997; Bertrand et al., 1999) and these invariants were found to be present in the active site cleft of MurD as observed in its 3-D structure. Similar results showing lack of activity with mutants of some of these invariant residues were reported for E. coli MurF (Eveland et al., 1997).

From the alignment of a large number of MurE sequences, a short sequence was identified as having two alternative conserved motifs characterized by the tetrapeptide sequences DNPR ( $m$-DAP recognition) and $\mathrm{D}(\mathrm{D} / \mathrm{N}) \mathrm{P}(\mathrm{N} / \mathrm{A})$ (L-lys recognition) (Dementin et al., 2001). Thermatoga maritima MurE, which was shown to incorporate L-lys to UDP-N-acetylmuramoyl dipeptide and also to add D-lys and $m$-DAP at $25 \%$ and $10 \%$ efficiencies respectively relative to Llysine, has the tetrapeptide sequence DDPR (Boniface et al., 2006). Staphylococcus aureus and Streptococcus pneumoniae MurE, which incorporate L-lys to UDP-N-acetylmuramoyl dipeptide, have DNPA and DDPN as the tetrapeptide sequence respectively.

Construction and characterization of mutants play a major role in understanding the function of specific residues in enzyme activity and substrate binding. This information along with the three dimensional structure of the protein will help in the rational design of inhibitors. In this study, truncation of the first $24 \mathrm{~N}$-terminal amino acids and site directed mutagenesis of some residues that are conserved in MurC, D, E, F, Mpl and FolC and some that are conserved in all MurE orthologs but not in all the other members of the superfamily were carried out in order to investigate their role in M. tuberculosis MurE (Mtb-MurE).

\section{RESULTS}

\section{Conservation of active site amino acid residues across the bacterial MurE}

The sequences of MurC, D, E and F from E. coli and MurE from T. maritima, $S$. aureus and $S$. pneumoniae were aligned with the MurE of $M$. tuberculosis using the ClustalW program and the final alignment was optimized manually (Fig. 1). The residues in boldface type can be divided into two categories. In the first category are the residues invariant across Mur synthetases, which are similar to those observed in studies on MurC and MurD (Bouhss et al., 1997, 1999). These are D71, K157 belonging to the ATP binding motif GXXGKT/S, E220, N243, H248, N344, N347, R377 and D392, numbered as per Mtb-MurE. Interestingly, N344, which was found to be conserved among the sequences aligned in this study, was not considered an invariant residue when MurD sequences were aligned (Bouhss et al., 1999) but was identified as an invariant residue when MurC sequences were aligned (Bouhss et al., 1997). N243 was identified as a further conserved residue during this alignment. In the second category, the DNPR motif conserved in all $m$-DAP adding enzymes, but not in all the other members of the Mur family, were looked at. The variation in this motif changes the substrate specificity from $m$-DAP to L-lysine in $S$. aureus and S. pneumoniae MurE. Intriguingly, T. maritima MurE adds Dlys and $m$-DAP in addition to L-lys although less efficiently.

\section{Generation and overexpression of Mtb-MurE mutants}

Mutagenesis of Mtb-MurE resulted in the replacement of specific residue N449 by aspartate, R451, K157, E220 and D392 residues by alanine; and the truncation of the first $24 \mathrm{~N}$ terminal amino acid residues. Analysis of the purified proteins by sodium dodecyl sulfate polyacrylamide gel electrophoresis (SDS-PAGE) (Fig. 2A) revealed that all the six mutant MurE proteins were overproduced to the same high level as the wild-type MurE and were stable in the host strain without significant proteolysis. Under optimum protein expression conditions around $3-5 \mathrm{mg}$ of protein was purified from one liter of $E$. coli culture.

\section{Enzymatic properties of the MurE mutants}

The wild type and mutant proteins were characterized in terms of their substrate binding, catalysis and substrate specificity using an ATPase activity assay. Initial velocity was measured in all the experiments to ensure the inorganic phosphate formed as a result of hydrolysis of ATP was well within the linear response of the reaction.

Specific activity for the mutant proteins using optimized conditions was found to be $0.03,0.07,0.11,0.16$ and $0.07 \mu \mathrm{moles} / \mathrm{min} / \mathrm{mg}$ for N449D, R451A, K157A, E220A and 


\begin{tabular}{|c|c|c|c|c|}
\hline & & & & \\
\hline TUMURE & $\begin{array}{l}71 \\
\text { DRTVTGVTLRAQDVSPG } 75\end{array}$ & \multicolumn{3}{|c|}{$\begin{array}{l}157 \\
\text { GITGTSGKTTTTYLVEAGLRAAGRVA } 175\end{array}$} \\
\hline COLIMURE & ----RALREMTIDSRVA 35 & \multicolumn{3}{|c|}{ GVTGTNGKTTTTQLLAQWSQLLGEIS 138} \\
\hline COLIMURD & ---ARGVTPRVMDTRMT 40 & \multicolumn{3}{|c|}{ AITGSNGKSTVTTLVGEMAKAAG--- 131} \\
\hline COLIMURC & VLANEGYQISGSDLAPN 54 & \multicolumn{3}{|c|}{ AIAGTHGKTTTTAMVSSIYAEAGLDP 148} \\
\hline COLIMURF & ---DITLDAVTTDTRKL 34 & \multicolumn{3}{|c|}{ ALTGSSGKTSVKEMTAAILSQCG--- 126} \\
\hline THEMAMURE & GVSNHSSKVKKGDLFIC 43 & \multicolumn{3}{|c|}{ GVTGTNGKTTTTMMIYHMLTSLGERG 132} \\
\hline STAAUMURE & ---EQQIDDITTDSRTA 33 & \multicolumn{3}{|c|}{ GVTGTNGKTSIATMIHLIORKLOKNS 132} \\
\hline STRPNMURE & ---KVIFDSISYDSRKV 45 & \multicolumn{3}{|c|}{ AFTGTKGKTTATYFAYNILSQGHRPA 140} \\
\hline YCTUMURE & $\begin{array}{l}220 \\
\text { TVVMEVSSHALALGRVD } 232\end{array}$ & \multicolumn{2}{|c|}{$\begin{array}{l}243 \\
\text { FAVGAFTMLSRDHLD--FHP } 253\end{array}$} & $\begin{array}{r}344347 \\
\text { PGRYNVAN } 347\end{array}$ \\
\hline ECOLIMURE & FCAMEVSSHGLVQHRVA 198 & \multicolumn{2}{|c|}{ FAASVFTYLSRDHLD--YHG 216} & MGAFNVSN 311 \\
\hline ECOLIMURD & LYVLELSSFQLETTS --168 & \multicolumn{2}{|c|}{ AVAATILNVTEDFMDR-YPF 190} & SGQHAYTM 272 \\
\hline ECOLIMURC & ----EADESDASFLHLQ 186 & \multicolumn{2}{|c|}{-MVAIVT\$IEADEMDT-YQG 205} & PGRHAALA 296 \\
\hline ECOLIMURF & YAVIELGANHQGEIAWT 170 & \multicolumn{2}{|c|}{ PEAALVNALAAAHLEG--FG 193} & PGRHAIAN 285 \\
\hline THEMAMURE & FFALEVSSHALVQQRVE 189 & \multicolumn{2}{|c|}{ FDVGIFTMISRDELD--FHG 210} & IGDFMAYN 296 \\
\hline STAAUMURE & SMTLEVSSHGLVLGRLR 189 & \multicolumn{2}{|c|}{ FDVAIFSNLTQDHLD--FHG 210} & VGKFNISN 304 \\
\hline STRPNMURE & HLIMEVSSQA------- 190 & \multicolumn{2}{|c|}{ FDVGVFIAITPDHIGPIEHP 220} & IGNFAQEA 301 \\
\hline IYCTUMURE & \multicolumn{2}{|c|}{\begin{tabular}{|c|}
377 \\
VPGRLEQID---------RGQGFLALVDYAHKP 397
\end{tabular}} & $\begin{array}{l}\text { A49 } 451 \\
\text { DNPRDE } 453\end{array}$ & \\
\hline ECOLIMURE & \multicolumn{2}{|c|}{ VCGRMEVFT---------APGKPTVVVDYAHTP 362} & \multirow{2}{*}{\multicolumn{2}{|c|}{ DNPRTE 419}} \\
\hline ECOLIMURD & \multicolumn{2}{|c|}{ LPHRFEVVL---------EHNGVRWINDSKAT- 322} & & \\
\hline ECOLIMURC & \multicolumn{2}{|c|}{ TGRRFDFLGEFPLEPVNGKSGTAMLVDDYGHHP 356} & \multicolumn{2}{|c|}{ PAGEAP 416} \\
\hline ECOLIMURF & \multicolumn{2}{|c|}{ VPGRLFPIQ-------LAENQLLIDDSYNA 335} & \multicolumn{2}{|c|}{ VLSVGK 392} \\
\hline THEMAMURE & \multicolumn{2}{|c|}{ VEGRFEVVRGA-------KKIGLNVVVDFAHSP 349} & \multicolumn{2}{|c|}{ DDPRGE 406} \\
\hline STAAUMURE & \multicolumn{2}{|c|}{ VEGRLEVLD-------PSLPIDLI IDYAHTA 355} & \multirow{2}{*}{\multicolumn{2}{|c|}{$\begin{array}{l}\text { DNPAND } 411 \\
\text { DPPNYF } 409\end{array}$}} \\
\hline STRPNMURE & \multicolumn{2}{|c|}{ VPGRMEVLT---------QKNGAKVFIDYAHNG 351} & & \\
\hline
\end{tabular}

Figure 1. Multiple sequence alignment of MurE protein. MurE from M. tuberculosis (MYCTU) was aligned with other homologous proteins from T. maritima (THEMA) (28\%), S. aureus (STAAU) (25\%), S. pneumoniae (STRPN) (25\%), and E. coli $(\mathrm{ECOLI})(37 \%)$ and similarly sequence alignment with other Mur ligases from E. coli MurD (16\%), MurC (17\%) and MurF (23\%). Only the clusters containing the conserved amino acid residues and the DNPR motif (in bold) for $m$-DAP adding and L-lys adding MurE of different organisms are shown. Percentage similarity is mentioned in parenthesis. The numbering refers to Mtb-MurE. The alignment function was performed by ClustalW. Hyphens represent a gap in the sequence.

D392A respectively (Fig. 2B) while the wild type showed $1.05 \mu \mathrm{moles} / \mathrm{min} / \mathrm{mg}$. The specific activity of the mutants was less than $15 \%$ of wild type, which nevertheless was still detectable above background. Deletion of the first 24 residues at the $\mathrm{N}$ terminus of MurE (MurEtr) did not lead to any significant variation in its activity compared to the wild type. The specific activity obtained for MurEtr was 0.9 umoles/ $\mathrm{min} / \mathrm{mg}$ protein.

\section{Effect on kinetic parameters}

Table 1 shows the kinetic parameters for endogenous substrates. Interestingly all the mutations have led to significant variations in apparent $K_{m}$ values for more than one substrate. For N449D, 4 fold increases in $K_{m}$ values were observed for ATP and $m$-DAP whereas a decrease was observed for UDP-MurNAc-L-ala-D-glu. Although there was no significant difference in $K_{m}$ value of ATP for R451A, the $K_{m}$ value for $m$-DAP was much larger and that for UDP-MurNAcL-ala-D-glu slightly lower. For K157A, a large variation in the $K_{m}$ values for UDP-MurNAc-L-ala-D-glu and $m$-DAP was observed. A major increase was observed for E220A and
D392A in their $K_{m}$ values for ATP with similar increase for UDP-MurNAc-L-ala-D-glu only with the E220A. No significant variations in $K_{m}$ for any of the three substrates, $V_{\max }$ and apparent $K_{\text {cat }}$ values were observed in case of MurEtr as compared to the wild type. A decrease in $V_{\text {max }}$ was observed for all the other mutants. This is in agreement with the calculated apparent $K_{\text {cat }}$ showing more than 10-fold decrease with all single residue replacement mutants except E220A, which showed about a 2 fold decrease.

\section{Substrate specificity of mutant proteins}

To better understand the interactions with mutated residues and the influence of the mutation on the substrate specificity, experiments with various analogs of each substrate were carried out (Tables 2-4). Interestingly wild type showed significant activity with DL-lanthionine similar to that of $\mathrm{m}$ DAP. N449D and MurEtr showed similar results to the wild type with all the nucleotides, UDP-MurNAc peptides and amino acids, except D-lys, which showed a slightly higher activity for N449D. A significant activity was observed in the presence of L-lys, D-lys, DL-ornithine and D-glu for R451A 

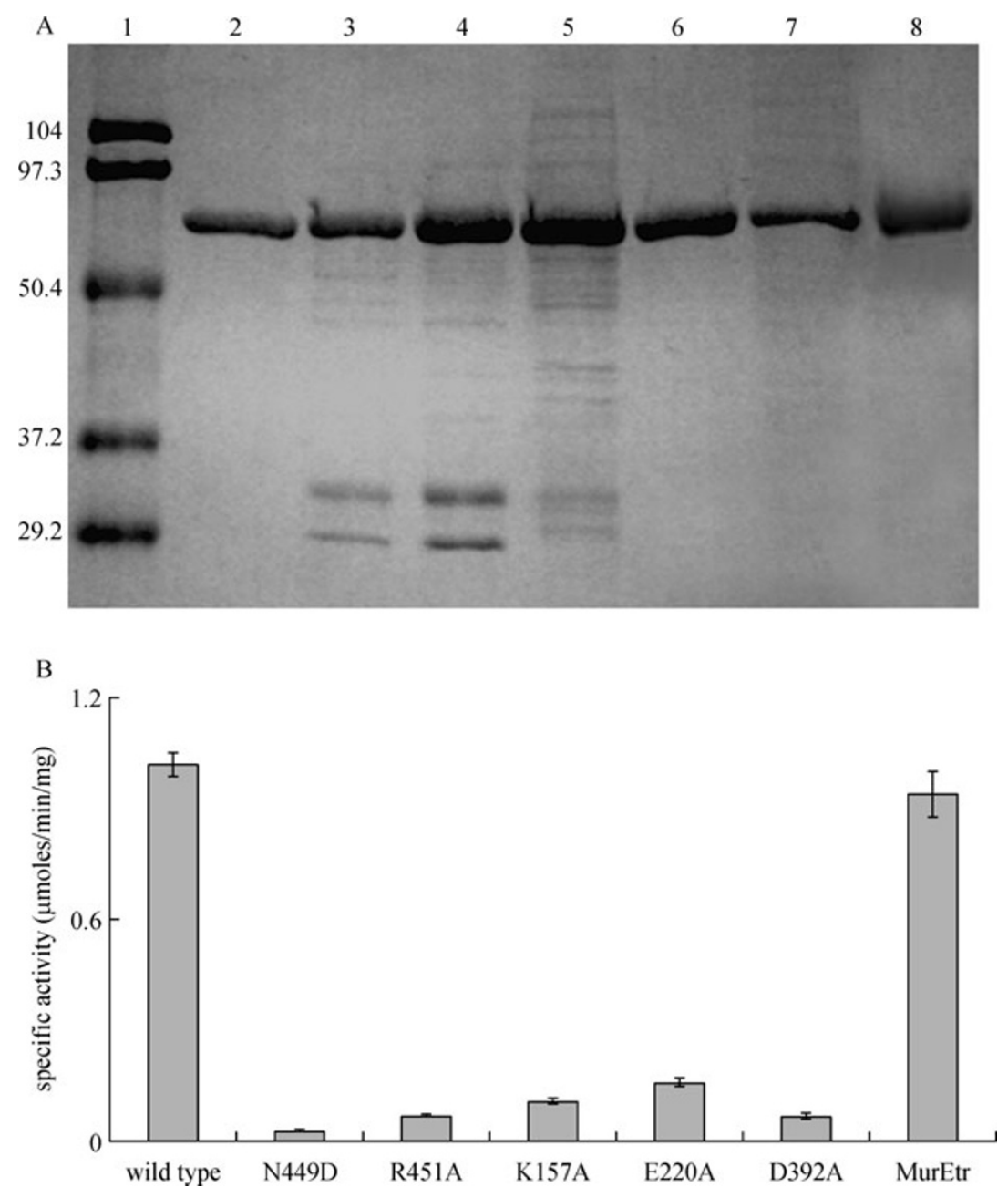

Figure 2. Comparison of wild type and mutant MurE from $M$. tuberculosis. (A) SDS-PAGE of purified fractions showing lane 1: protein molecular mass markers, individual molecular mass in $\mathrm{kDa}$ is indicated to the left; lane 2: wild type; lane 3: N449D; lane 4: R451A; lane 5: K157A; lane 6: E220A; lane 7: D392A; lane 8: MurEtr. In all cases 4-6 $\mu \mathrm{g}$ of protein loaded on the gel. (B) Comparison of specific activity of wild type MurE with mutant proteins. The reaction consisted of Bis-tris-propane- $\mathrm{HCl}(\mathrm{pH} 8.5), \mathrm{MgCl}_{2}, \mathrm{ATP}, \mathrm{UDP}-$ MurNAc dipeptide, $m$-DAP and enzymes at the concentration described in the experimental procedures. In all cases, enzymes were omitted in the control reactions. Y-axis represents the amount of Pi released in $\mu \mathrm{moles} / \mathrm{min} / \mathrm{mg}$ of protein. Error bars indicate the standard deviation. SDS-PAGE: sodium dodecyl sulfate polyacrylamide gel electrophoresis.

Table 1 Kinetic parameters of wild type and mutant MurE proteins for endogenous substrates

\begin{tabular}{lccccc}
\hline protein & $\begin{array}{c}\text { app } K_{m}{ }^{\text {ATP }} \\
(\mathrm{mM})\end{array}$ & $\begin{array}{c}\text { app } K_{m} \text { UMAG } \\
(\mathrm{mM})\end{array}$ & $\begin{array}{c}\mathrm{app} K_{m}{ }^{m-D A P} \\
(\mathrm{mM})\end{array}$ & $\begin{array}{c}\text { app } V_{\max }(\mathrm{nmol} / \mathrm{min}) \\
{[\mathrm{mg} \text { of protein }]^{-1}}\end{array}$ & $\begin{array}{c}\text { app } K_{\text {cat }} \\
\left(\mathrm{min}^{-1}\right)\end{array}$ \\
\hline wild type & $0.017 \pm 0.0007$ & $0.024 \pm 0.0015$ & $0.078 \pm 0.004$ & $790 \pm 28$ & $78 \pm 2$ \\
N449D & $0.060 \pm 0.005$ & $0.007 \pm 0.0003$ & $0.324 \pm 0.001$ & $69 \pm 3$ & $3 \pm 0.2$ \\
R451A & $0.015 \pm 0.001$ & $0.005 \pm 0.0001$ & $0.473 \pm 0.005$ & $71 \pm 4$ & $4 \pm 0.1$ \\
K157A & $0.008 \pm 0.0006$ & $<0.005$ & $0.012 \pm 0.001$ & $90 \pm 7$ & $5 \pm 0.4$ \\
E220A & $0.123 \pm 0.01$ & $0.148 \pm 0.005$ & $0.052 \pm 0.002$ & $320 \pm 20$ & $34 \pm 2$ \\
D392A & $0.100 \pm 0.008$ & $0.016 \pm 0.0006$ & $0.113 \pm 0.008$ & $70 \pm 7$ & $6 \pm 0.3$ \\
MurEtr & $0.021 \pm 0.004$ & $0.019 \pm 0.0022$ & $0.065 \pm 0.0016$ & $709 \pm 0.5$ & $67 \pm 1.2$ \\
\hline
\end{tabular}

app: apparent; UMAG: UDP-MurNAc-L-ala-D-glu 
Table 2 Substrate specificity of different amino acids with wild type and mutant proteins

\begin{tabular}{|c|c|c|c|c|c|c|c|}
\hline \multirow[b]{2}{*}{ amino acid } & \multicolumn{7}{|c|}{ relative activity (\%) ${ }^{a}$} \\
\hline & wild type & N449D & R451A & K157A & E220A & D392A & MurEtr \\
\hline$m$-DAP & $100 \pm 7.5$ & $100 \pm 6.8$ & $100 \pm 8.9$ & $100 \pm 9.2$ & $100 \pm 9.5$ & $100 \pm 5.6$ & $100 \pm 1.2$ \\
\hline DL-lanthionine & $82 \pm 5.1$ & $76 \pm 4.3$ & $78 \pm 3.8$ & $100 \pm 8.7$ & $83 \pm 6.3$ & $82 \pm 4.5$ & $97 \pm 2.4$ \\
\hline L-cystathionine & $12 \pm 0.6$ & 0 & $34 \pm 3$ & $100 \pm 9.1$ & $100 \pm 7.6$ & $81 \pm 5.7$ & $3 \pm 0.8$ \\
\hline L-lys & $7 \pm 0.6$ & $2 \pm 0.12$ & $45 \pm 3.6$ & $100 \pm 8.6$ & $100 \pm 7.9$ & $84 \pm 6.8$ & $9 \pm 0.43$ \\
\hline D-lys & $9 \pm 0.35$ & $37 \pm 3$ & $62 \pm 5$ & $100 \pm 6.9$ & $100 \pm 8.8$ & $78 \pm 6.1$ & $7 \pm 0.55$ \\
\hline L-ala & $12 \pm 0.82$ & 0 & 0 & $100 \pm 8.6$ & $100 \pm 7.7$ & $99 \pm 6.8$ & $3 \pm 0.21$ \\
\hline D-glu & $13 \pm 0.42$ & $6 \pm 0.4$ & $100 \pm 5.9$ & $92 \pm 6.8$ & $95 \pm 7.2$ & $88 \pm 5.8$ & $3 \pm 0.36$ \\
\hline D-ala-D-ala & $5 \pm 0.3$ & $1 \pm 0.07$ & $18 \pm 1.8$ & $100 \pm 7.2$ & $98 \pm 8.3$ & $88 \pm 6.9$ & $1 \pm 0.67$ \\
\hline DI-ORNITHINE & $8 \pm 0.72$ & $8 \pm 0.56$ & $8 \pm 6.9$ & $85 \pm 7.2$ & $90 \pm 5.7$ & $86 \pm 7$ & $2 \pm 0.15$ \\
\hline
\end{tabular}

${ }^{a}$ Activity with $m$-DAP was used as $100 \%$ for each protein. $m$-DAP: meso-diaminopimelic acid.

Table 3 Substrate specificity of different uridine sugar precursors with wild type and mutant proteins

\begin{tabular}{lccccccc}
\hline & \multicolumn{7}{c}{ relative activity (\%) } \\
\cline { 2 - 8 } nucleotides & wild type & N449D & R451A & K157A & E220A & D392A & MurEtr \\
\hline ATP & $100 \pm 6.8$ & $100 \pm 8.1$ & $100 \pm 7.4$ & $100 \pm 6.7$ & $100 \pm 5.5$ & $100 \pm 4.8$ & $100 \pm 5.4$ \\
GTP & $30 \pm 2.5$ & 0 & $17 \pm 1.1$ & $98 \pm 3.8$ & $100 \pm 6.2$ & $100 \pm 7.7$ & $30 \pm 1.8$ \\
UTP & $17 \pm 1.5$ & 0 & $5 \pm 0.35$ & $100 \pm 6$ & $92 \pm 5.8$ & $99 \pm 6.8$ & $5 \pm 0.55$ \\
UTP & $14 \pm 0.98$ & 0 & 0 & $83 \pm 7.1$ & $82 \pm 5.7$ & $96 \pm 4.6$ & $6 \pm 0.21$ \\
\hline
\end{tabular}

activity with ATP was used as $100 \%$ for each protein.

Table 4 Substrate specificity of different nucleotides with wild type and mutant proteins

\begin{tabular}{|c|c|c|c|c|c|c|c|}
\hline \multirow[b]{2}{*}{ UDP-MurNAc peptides } & \multicolumn{7}{|c|}{ relative activity $(\%)^{\mathrm{a}}$} \\
\hline & wild type & N449D & R451A & K157A & E220A & D392A & MurEtr \\
\hline UDP-MurNAc dipeptide & $100 \pm 4.7$ & $100 \pm 6.9$ & $100 \pm 7.5$ & $100 \pm 5.8$ & $100 \pm 7.7$ & $100 \pm 4.7$ & $100 \pm 5.4$ \\
\hline MurNAc & $14 \pm 0.87$ & $14 \pm 0.94$ & $17 \pm 0.77$ & $98 \pm 6.6$ & $100 \pm 5.8$ & $100 \pm 5.8$ & $5 \pm 0.68$ \\
\hline UDP-MurNAc tripeptide & $8 \pm 0.73$ & $20 \pm 1.1$ & $3 \pm 0.2$ & $96 \pm 6.8$ & $93 \pm 5.7$ & $98 \pm 8.8$ & $5 \pm 0.37$ \\
\hline UDP-MurNAc pentapeptide & $15 \pm 0.97$ & $16 \pm 0.88$ & $18 \pm 0.67$ & $97 \pm 7.4$ & $99 \pm 6.8$ & $83 \pm 4.6$ & $4 \pm 0.11$ \\
\hline
\end{tabular}

${ }^{a}$ Activity with UDP-MurNAc dipeptide was used as $100 \%$ for each protein.

with similar activities as wild type for all the nucleotides and UDP-MurNAc peptides tested. Surprisingly, K157A, E220A and D392A showed very high activity with all the amino acids, uridine sugar precursors and nucleotides tested compared to wild type and moreover, reaching up to $100 \%$ of the standard assay in many cases.

\section{Effect of ATP, $m$-DAP and UDP-MurNAc-L-ala-D-glu on ATP hydrolysis}

The substrate specificity experiments led us to investigate further the effect of ATP alone and in combination with mesodiaminopimelic acid ( $m$-DAP) and UDP-MurNAc-L-ala-D-glu (Table 5). Wild type, N449D, R451A and MurEtr showed the lowest absorbance in the presence of only ATP and enzyme, which does not change much in the presence of $m$-DAP, whereas absorbance increased slightly in the presence of uridine sugar precursor similar to all substrates without enzyme. K157A, E220A and D392A showed significant ATP hydrolysis compared to wild type, N449D, R451A and MurEtr in the presence of only ATP and enzyme, in the absence of other substrates. This activity was higher than the control reaction but similar to that in the presence of $m$-DAP. The ATP hydrolysis activity further increased significantly in the presence of uridine sugar precursors to a similar level to that of the enzyme reaction with all the three substrates.

\section{Crystal structure of MurE/ADP/dipeptide ternary complex}

The ternary complex structure (PDB: 2xja) shows clear density for ADP with very little other difference from the MurE/dipeptide complex (PDB: 2wtz). The refinement statis- 
Table 5 Activity in the presence of different components of the MurE reaction with the wild type and mutant proteins

\begin{tabular}{|c|c|c|c|c|c|c|c|}
\hline \multirow[b]{2}{*}{ different conditions } & \multicolumn{7}{|c|}{ relative absorbance $(\%)^{a}$} \\
\hline & wild type & N449D & R451A & K157A & E220A & D392A & MurEtr \\
\hline ATP + enzyme & $29 \pm 2.4$ & $37 \pm 3.6$ & $39 \pm 2.7$ & $77 \pm 4.1$ & $66 \pm 3.8$ & $70 \pm 4.2$ & $19 \pm 1.3$ \\
\hline $\begin{array}{l}\text { ATP + enzyme + UDP MurNAc } \\
\text { dipeptide }\end{array}$ & $52 \pm 3.2$ & $65 \pm 4.2$ & $64 \pm 3.8$ & $85 \pm 4.4$ & $89 \pm 6.2$ & $98 \pm 7.3$ & $36 \pm 2.6$ \\
\hline ATP + enzyme $+m$-DAP & $28 \pm 2.1$ & $34 \pm 2.7$ & $38 \pm 1.9$ & $73 \pm 4.5$ & $66 \pm 3.8$ & $67 \pm 2.4$ & $21 \pm 2.0$ \\
\hline $\begin{array}{l}\text { ATP + UDP MurNAc dipeptide + } \\
m \text {-DAP }\end{array}$ & $48 \pm 1.4$ & $59 \pm 2.5$ & $58 \pm 3.1$ & $55 \pm 1.9$ & $62 \pm 2.3$ & $66 \pm 3.2$ & $38 \pm 3.5$ \\
\hline $\begin{array}{l}\text { ATP + enzyme + UDP MurNAc } \\
\text { dipeptide + } m \text {-DAP }\end{array}$ & $100 \pm 5.4$ & $100 \pm 4.9$ & $100 \pm 5.2$ & $100 \pm 4.6$ & $100 \pm 6.2$ & $100 \pm 4.8$ & $100 \pm 6.6$ \\
\hline
\end{tabular}

tics are in Table 6. The refined structure is depicted in Figs. 3 and 4 showing binding of ADP in an analogous position to that in MurD.

\begin{tabular}{lc} 
Table 6 & Data collection and refinement statistics \\
\hline ligand combination & UMAG + ADP \\
space group & $\mathrm{P} 1$ \\
chains per unit cell & 4 \\
completeness $(\%)$ & 95.9 \\
multiplicity & 3.7 \\
average $\mathrm{R}_{\text {merge }}$ & 0.12 \\
average $/ / \sigma$ & 9.1 \\
outer shell resolution $(\AA)$ & $3.00-3.16$ \\
outer shell $\mathrm{R}_{\text {merge }}$ & 0.57 \\
outer shell $/ / \sigma$ & 2.6 \\
refined $\mathrm{R}$ factor & 0.189 \\
final $\mathrm{R}_{\text {free }}$ & 0.258 \\
\hline
\end{tabular}

\section{DISCUSSION}

Structural analysis of wild type Mtb-MurE, from our previous study, had revealed that the first $24 \mathrm{~N}$-terminal amino acid residues may not be involved in the active site structure of MurE (Basavannacharya et al., 2010); hence, a truncated version of wild type MurE was generated. The truncated MurE mutant showed activities similar to the wild type MurE, providing further support that this deletion did not affect the activity nor the substrate specificity of the recombinant enzyme.

The $m$-DAP of the peptidoglycan monomer unit plays a pivotal role in the integrity of the peptidoglycan as it is directly involved in peptide cross-linkages (Glauner et al., 1988). mDAP is used in various transpeptidation reactions during the later stages of peptidoglycan biosynthesis, which leads to the formation of $\mathrm{D}$-ala- $>m$-DAP and $m$-DAP- $>m$-DAP cross linkages (Glauner et al., 1988; Nanninga, 1991). Interestingly, peptidoglycan of some bacterial species contain 3-hydroxy $m$-DAP or lanthionine as a natural substitute for $m$-DAP (Inoue et al., 1979; Vasstrand et al., 1980; Kawamoto et al., 1981). It has been shown that in $m$-DAP auxotrophs, L-allocystathionine (95\%) and $m$-lanthionine (93\%), sulfur containing $m$-DAP analogs, were incorporated into the peptidoglycan of $E$. coli in in vivo conditions. These are shown to be good substrates in addition to D-allo-cystathionine for the $E$. coli enzyme (Mengin-Lecreulx et al., 1994) concluding that MurE was the cystathionine and lanthionine adding enzyme in vivo. Mycobacterium smegmatis has been shown to incorporate lanthionine in m-DAP auxotrophs. Even though cells grow, they do not produce normal peptidoglycan. The replacement of $m$-DAP with lanthionine but not cystathionine suggested that the peptidoglycan biosynthesis in mycobacteria may have more constraints on peptide composition than in E. coli (Consaul et al., 2005). Interestingly, in the case of $M$. tuberculosis wild type MurE showed a similar activity with DL-lanthionine as $m$-DAP, but much lower activity with Lcystathionine or any of the other amino acids. This result is consistent with that observed in the mutant strains of $E$. coli (Mengin-Lecreulx et al., 1994) and M. smegmatis (Consaul et al., 2005) demonstrating MurE specificity for the meso isomer.

Asparagine 449, belonging to a well-known consensus DNPR motif (Gordon et al., 2001) conserved in $m$-DAP adding enzymes, was mutated to aspartate in N449D to investigate its role in substrate binding and incorporation of substrates, particularly $m$-DAP. The $K_{m}$ values for $m$-DAP and ATP were increased by 4 fold whereas for UDP-MurNAc-Lala-D-glu, there was no significant change. These results suggested that this residue is involved in $m$-DAP and ATP binding. Further evidence comes from the structure of $E$. coli MurE with the product complex (Gordon et al., 2001), where the amino group of amide side chain of Asn414 forms a hydrogen bond with the carboxylic group of $m$-DAP at the 


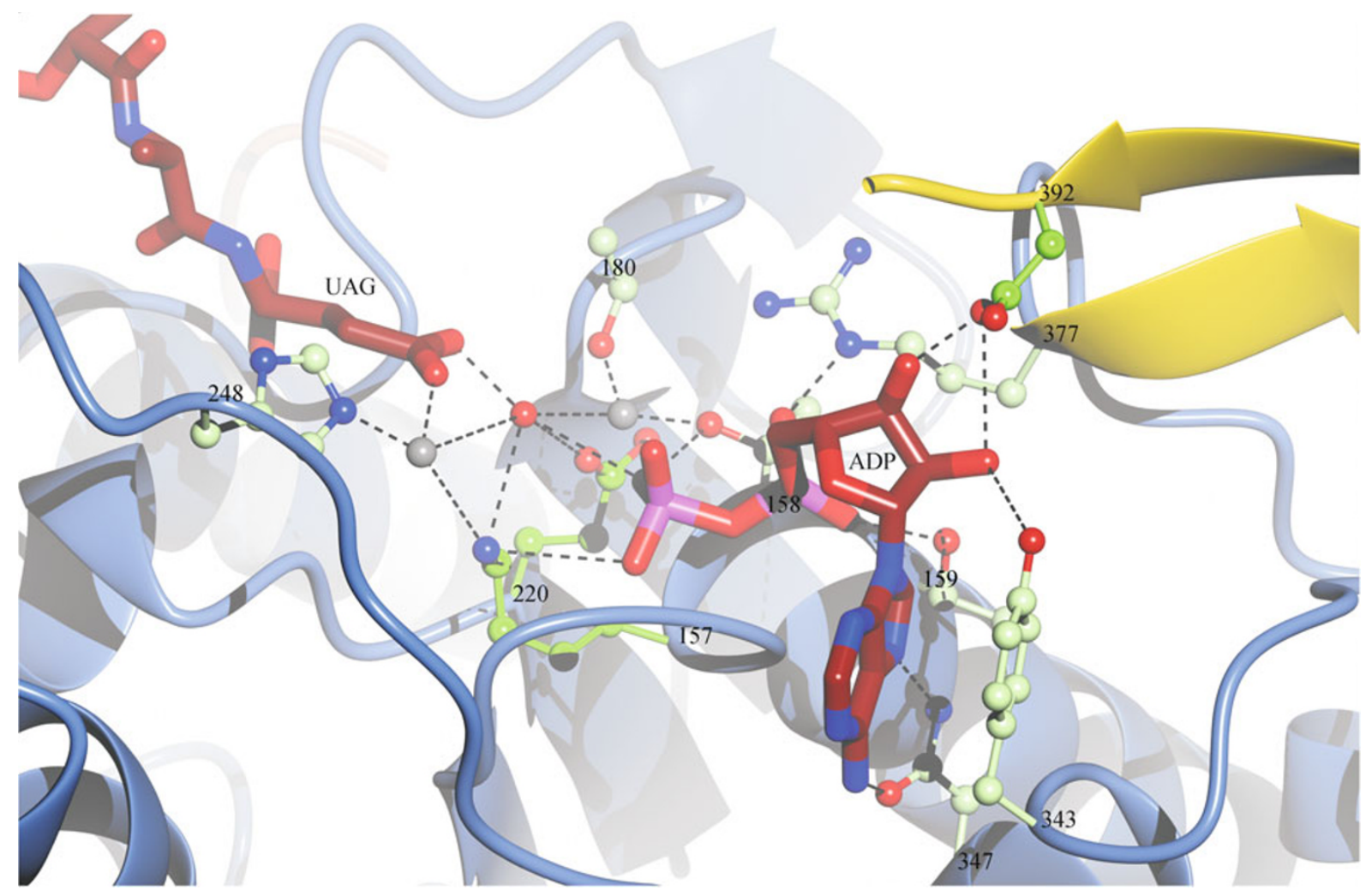

Figure 3. Three dimensional structure of Mtb-MurE/ADP/Mg ${ }^{2+}$ complex. A close up of the active site of $M$. tuberculosis MurE showing the 3 mutated residues close to ADP in dark green and other side chains interacting with ADP in light green labeled with residue numbers at $\mathrm{C}_{\text {alpha. }}$. ADP and UAG are shown as tan cylinders, magnesium as gray spheres and a highly ordered water as a red sphere. Hydrogen bonds are shown as dotted lines. Domain 1 is in coral, domain 2 is in blue and domain 3 is in yellow and truncated at residue 394 .

D-center (Fig. 5). This interaction gets disrupted when the equivalent Asn449 in Mtb-MurE was converted to aspartate and loses the affinity for the substrate. But how this mutation is involved in ATP binding is difficult to explain. A high degree of reduction, by 35 fold in the specific activity, was reflected by 26 fold decrease in the turnover rate. The N449D mutation results in DDPR motif present in T. maritima, a thermophilic bacterium which shows $25 \%$ and $10 \%$ efficiency of incorporation of D-lys and $m$-DAP relative to L-lys (Boniface et al., 2006). However, this mutation neither abolishes the incorporation of $m$-DAP, nor does it show any activity with L-lys. This is not surprising as the D-carbon (distal recognition site) of D-lys is involved in interaction with the residues (Glu468, Gly464, Asp413, Asn414 and Arg416) of E. coli MurE (Fig. 5) and the $L$-carbon gets acylated on its $\varepsilon$-amino group similar to $m$-DAP (Gordon et al., 2001). Similar observations were made in E. coli in vivo (Mengin-Lecreulx et al., 1994).

Another residue arginine 451, also part of the DNPR motif, is also conserved in organisms incorporating $m$-DAP at the third position of stem peptide of the UDP-MurNAc pentapeptide. This residue is believed to be responsible for determining the specificity of $m$-DAP incorporation into the peptidoglycan in E. coli (Gordon et al., 2001). The R451A mutant was made to investigate further its role in substrate specificity. There was a 6 fold increase observed in $K_{m}$ value for $m$-DAP whereas a 5 fold decrease was seen for UDP-MurNAc-L-ala-D-glu and no difference for ATP. These results support the involvement of this residue in $m$-DAP incorporation. Evidence from the $E$. coli MurE product structure strongly supports this role for this residue. Two amino groups from guanidinium side chain of Arg416 are involved in hydrogen bonding with carboxylic group of $m$-DAP at the $\mathrm{D}$ center as well as at the $\mathrm{L}$ center (Fig. 5) (Gordon et al., 2001). The corresponding arginine at position 451 in Mtb-MurE loses these interactions when replaced by alanine, thereby showing a reduced affinity for $m$ DAP, but the negative role of this residue in UDP-MurNAc-Lala-D-glu binding as seen by decrease in the $K_{m}$ value is difficult to understand. R451A also showed loss of activity by 15 -fold which agrees well with the 20 -fold reduction in the turnover rate of this enzyme. The resulting mutation has the DNPA sequence which is present in S. aureus. This Grampositive organism incorporates L-lys at the third position of the stem peptide in the peptidoglycan precursor. This mutation in MurE of M. tuberculosis did not abolish the incorporation of 


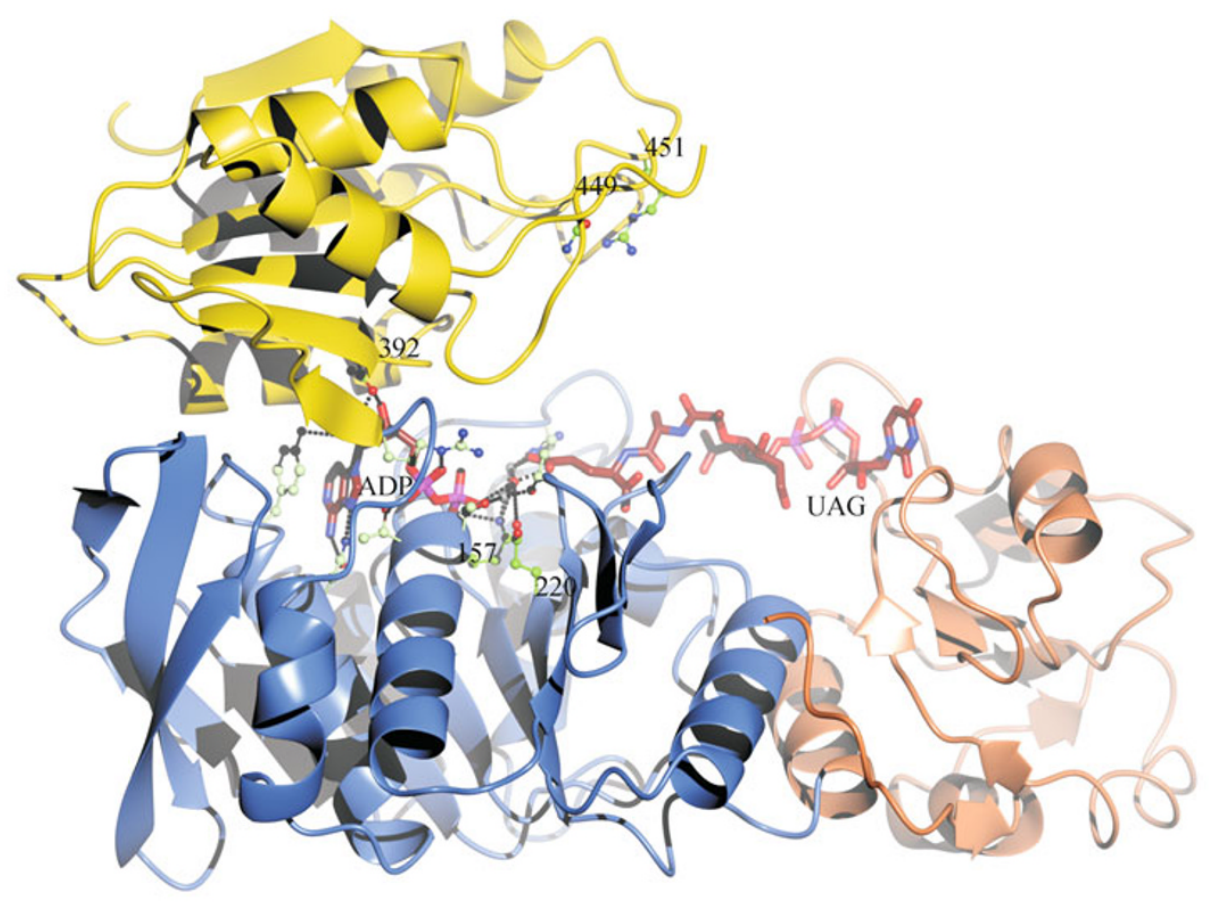

Figure 4. Location of the residues subjected to mutagenesis. Amino acid residues that were mutated are indicted by ball and stick side chains labeled by residue number in green, in the crystal structure of MurE of $M$. tuberculosis colored by domains (1 in coral, 2 in blue, and 3 in yellow). The ligands UAG and ADP are shown by tan cylinder models, $\mathrm{and}_{\mathrm{Mg}^{2+}}$ is shown as gray spheres.

$m$-DAP, instead additionally it showed significant activity with L-lys. It also showed substantial activity with DL-lanthionine, D-lys, D-glu and DL-ornithine.

Lysine at position 157 belongs to a well-known consensus ATP binding site (Walker et al., 1982; van der Wolk et al., 1995). Surprisingly, the $K_{m}$ value of K157A for ATP was reduced 2 fold. In the three dimensional structure of MtbMurE/ADP $/ \mathrm{Mg}^{2+}$ complex (Fig. 3), this lysine forms interacting bonds through its amino group with the $\beta$-phosphate of ADP similar to $E$. coli MurD at position 115 (Bertrand et al., 1999). It is clear from the structural data, that the interaction with $\varepsilon$ amino group is lost when lysine is replaced by alanine. The same mutation showed apparent stronger affinity for the other two substrates UDP-MurNAc-L-ala-D-glu and $m$-DAP indicated by their reduced $K_{m}$ value. This is in contrast to the data observed for mutation of the equivalent residues in $E$. coli MurC and MurD which showed an increase in $K_{m}$ for ATP and uridine sugar precursor (Bouhss et al., 1997; Bouhss et al., 1999). For K157A, the turnover rate was 16-fold lower than that of wild type with 10-fold reduction in its enzyme activity strongly suggesting its role in catalysis. This is in agreement with the results with MurC and MurD (Bouhss et al., 1997; Bouhss et al., 1999).

The $K_{m}$ value for E220A was significantly increased by 7 fold for ATP giving a clue that this residue has a crucial role to play in ATP binding. This is predicted from the fact that this residue belongs to the second ATP binding motif (Walker et al., 1982). The residue is conserved across various ATP binding proteins including FolC (Bognar et al., 1987). This residue is generally involved in the chelation of the ATP- $\mathrm{Mg}^{2+}$ complexes (Walker et al., 1982; Glaser et al., 1991; Story and Steitz, 1992; Mitchell and Oliver, 1993). The Mtb-MurE/ADP/ $\mathrm{Mg}^{2+}$ complex reveals that E220 is involved in interactions with $\mathrm{Mg}^{2+}$ which in turn binds ADP (Fig. 3) similar to the corresponding E157 in E. coli MurD binding to ATP through $\mathrm{Mg}^{2+}$ (Bertrand et al., 1999). When this interaction is disrupted by the mutation, the affinity of ATP binding is lowered. The $K_{m}$ value for UDP-MurNAc-L-ala-D-glu was 6 fold higher whereas $m$-DAP showed an insignificant decrease in $K_{m}$ value. A similar result showing a drastic increase in $K_{m}$ for the uridine sugar precursor was observed for the analogous mutation of MurD in E. coli (Bouhss et al., 1999). E220A demonstrated a specific activity 6 fold less with 2 fold decrease in turnover rate. This is in contrast to the results obtained with E. coli MurC and MurD (Bouhss et al., 1997; Bouhss et al., 1999) and MurF (Eveland et al., 1997) where there was a greater loss of activity with these proteins caused by the analogous mutation.

In D392A where aspartate 392 is replaced by alanine, a 5 fold increase in the $K_{m}$ value was observed for ATP whereas 


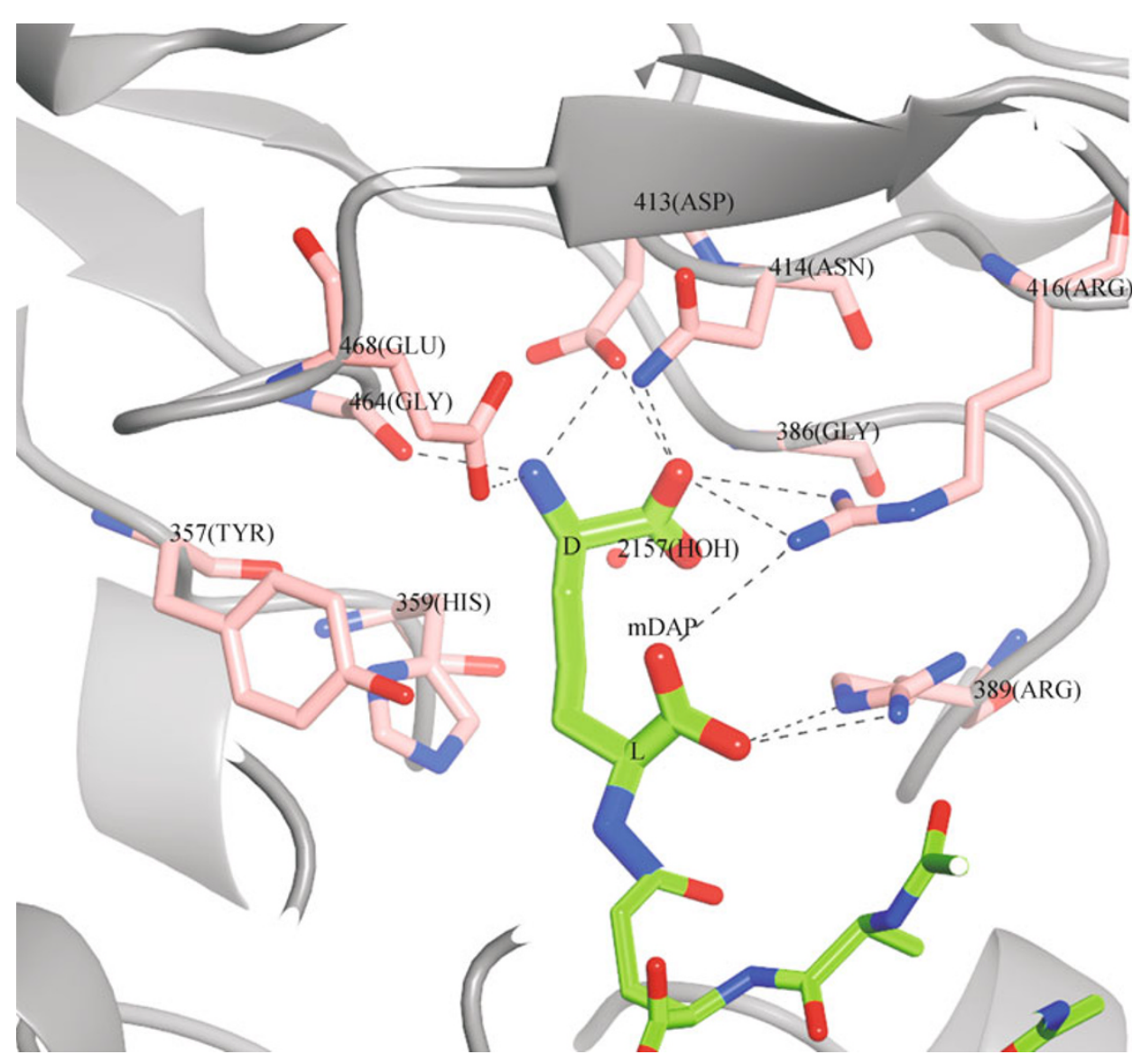

Figure 5. Structure of the E. coli MurE product (1e8c). The product UDP-N-acetylmuramoyl-L-alanyl-D-gamma-glutamyl-meso2,6-diamino-heptanedioate (UMT) is shown in green and contacting side chains in pink. Hydrogen bonds are shown as dotted lines.

for other substrates, it was of the same order of magnitude as that of wild type. In the three dimensional structure of Mtb-MurE/ADP/Mg ${ }^{2+}$ complex (Fig. 3), this residue has been shown to make interactions with the ribose moiety of ADP through its carboxylate oxygens, similar to the equivalent residue Asp317 in E. coli MurD (Bertrand et al., 1999). When this residue is mutated to alanine the mutation results in loss of these interactions thereby losing the affinity for ATP. In MurD mutant of $E$. coli, a strong increase in the $K_{m}$ value for ATP and uridine sugar precursor was detected (Bouhss et al., 1999). The D392 residue appears to play a crucial role in catalysis as there was a reduction in the specific activity and turnover number by 15 -fold. This is in agreement with that obtained in analogous mutants of E. coli MurC and MurD (Bouhss et al., 1997; Bouhss et al., 1999).

K157A, E220A and D392A showed significant activity with all the amino acids, nucleotides and uridine sugars tested to a level comparable to that of $m$-DAP, ATP and UDP-MurNAc-Lala-D-glu respectively, indicating that these mutations may have caused ATP hydrolysis to be uncoupled from the enzyme reaction.
Further investigation was done only in the presence of ATP in order to gain more understanding of the above results. The results found from this experiment were intriguing. There seem to be significant ATP hydrolysis taking place with K157A, E220A and D392A even in the absence of other substrates contrary to that of wild type, N449D, R451A and MurEtr. The hydrolysis rate of the mutants remained same after the addition of $m$-DAP, whereas it significantly increased in the presence of uridine sugar precursor. The binding of MurNAc, the common moiety in all the uridine sugars tested, seem to cause more ATP hydrolysis by an allosteric effect. The high rate of phosphate release with different amino acids, nucleotides and uridine sugar precursors with K157A, E220A and D392A is due to the uncoupled hydrolysis of ATP by the enzyme. ATPase contamination in the preparations of these mutants is unlikely as the purification was carried out under identical conditions for these mutants as that for wild type, N449D, R451A and MurEtr. The baseline reactions with only the mutant proteins did not show any phosphate contamination (Table 7). It is well established that the ATPase activity is coupled with ligase activity for the wild type protein 
Table 7 Absorbance of individual assay components at $635 \mathrm{~nm}$

\begin{tabular}{lc}
\hline different conditions & $\mathrm{A}_{635 \mathrm{~nm}}$ \\
\hline ATP alone & 0.53 \\
UDP-MurNAc dipeptide alone & 0.47 \\
m-DAP alone & 0.29 \\
wild type & 0.29 \\
N449D & 0.28 \\
R451A & 0.28 \\
K157A & 0.27 \\
E220A & 0.29 \\
D392A & 0.30 \\
MurEtr & 0.26 \\
\hline
\end{tabular}

(Basavannacharya et al., 2010). Hence, the residues involved in these mutations are likely responsible for coupling the ATPase activity with the ligase activity.

The findings in this study contribute significantly to the understanding of the residues essential for catalytic activity, substrate binding and specificity of MurE of M. tuberculosis. In particular, it identifies the residues that couple hydrolysis of ATP to the reaction. These results along with the three dimensional structure of Mtb-MurE will aid in the development of inhibitors targeting the drug-resistant strains of $M$. tuberculosis.

\section{MATERIALS AND METHODS}

\section{Strains, plasmids and growth conditions}

E. coli DH5a (Bioline, UK) and BL21 (DE3) (Promega Corporation) strains were used in this study. Oligonucleotide primers were synthesized by MWG Biotech, Germany. Restriction enzymes were obtained from New England Biolabs, UK. All other media and chemicals were purchased from Sigma unless otherwise mentioned. LB broth and agar media were used for growing cells. Growth was monitored by measuring the culture absorbance at $600 \mathrm{~nm}$. Standard procedures for endonuclease digestion, ligation and agarose electrophoresis were used (Sambrook and Russell, 2001).

\section{Generation of Mtb-MurE mutants}

pET28b-murE (pSBC1), a plasmid harbouring the wild type Mtb-murE gene generated in a previous study (Basavannacharya et al., 2010) was used as the template for the mutagenic PCR. Site-directed mutagenesis was carried out using the QuikChange ${ }^{\circledR}$ Lightning sitedirected mutagenesis kit (Strategene) according to the manufacturer's instructions. Truncation of the first 24 amino acid residues at the $\mathrm{N}$-terminal end ( 72 base pairs) was based on similar principle as site-directed mutagenesis. All primers used in this mutagenesis study are described in Table 8. DNA sequence analysis was done to confirm that mutation has taken place at the desired positions. The plasmids with amino acid residue mutations were designated as pSBC1K157A, pSBC1E220A, pSBC1D392A, pSBC1N449D and pSBC1R451A, and that with the truncated MurE was termed as pSBC1Etr.

\section{Expression and purification of wild type and mutant MurE proteins}

The expression and purification of wild type and mutant proteins was carried out as described earlier (Basavannacharya et al., 2010), with slight modifications. On harvesting the cultures, the cell pellets were washed and resuspended in ice-cold buffer $\mathrm{A}$ containing $0.02 \mathrm{M}$ Bistris propane $\cdot \mathrm{HCl}(\mathrm{pH} 8.5), 0.1 \mathrm{M} \mathrm{NaCl}$ and $0.03 \mathrm{M}$ imidazole. Once the bound proteins were eluted from $\mathrm{Ni}^{2+}-\mathrm{NTA}$ resin with $100 \mathrm{mM}$ imidazole in buffer $\mathrm{A}$, they were diluted in storage buffer [0.02 M Bistris propane $\cdot \mathrm{HCl}(\mathrm{pH} 8.5)$ and $0.1 \mathrm{M} \mathrm{NaCl}$ ] and reconcentrated to remove imidazole. Protein concentration was estimated by Bradford assay (Bradford, 1976) using bovine serum albumin as a standard and analyzed by SDS-PAGE (Laemmli, 1970). Glycerol was added to a final concentration of $10 \%(\mathrm{~V} / \mathrm{V})$ and the proteins were stored in aliquots at $-80^{\circ} \mathrm{C}$. The resulting mutant proteins were designated as N449D, R451A, K157A, E220A, D392A and MurEtr.

\section{MurE activity assay}

MurE enzyme activity was assayed as described previously (Basavannacharya et al., 2010), by measuring the release of inorganic phosphate following ATP hydrolysis using Pi ColorLock Gold kit (Innova Biosciences). The enzyme activity assay was

Table 8 Mutagenic oligonucleotides

\begin{tabular}{lll}
\hline plasmid & mutation $^{\mathrm{a}}$ & oligonucleotide $^{\mathrm{b}}$ \\
\hline pSBC1N449D & N449D & 5'-GTCACCGACGACGACCCGCGTGACGAAG-3' \\
pSBC1R451A & R451A & 5'-GACGACAACCCGGCTGACGAAGATCCCACG-3' \\
pSBC1K157A & K157A & 5'-AACGTCCGGCGCGACCACCACCACCTATC-3' \\
pSBC1E220A & E220A & 5'-ACACCGTGGTCATGGCGGTGTCCAGCCACG-3' \\
pSBC1D392A & D392A & 5'-CGCTGGTCGCCTACGCGCAC-3' \\
pSBC1MurEtr & 24 residues & 5'-CCTGGTGCCGCGCGGCAGCCAggcttgcgccccaacgccgtc-3'c
\end{tabular}

\footnotetext{
${ }^{a}$ Amino acids are represented by their one-letter abbreviation and the number indicates the location of the mutated residue in the amino acid sequence of MurE from $M$. tuberculosis.

${ }^{\mathrm{b}}$ Mutations of the murE gene sequence that have been introduced in the oligonucleotides are indicated in bold.

${ }^{c}$ Primers for truncation of $\mathrm{N}$-terminal residues of murE with bases in upper and lower case representing sequence flanking the 72-base-pair region to be truncated.
} 
performed in a final volume of $50 \mu \mathrm{L}$ in the presence of $25 \mathrm{mM}$ Bis-tris propane $\cdot \mathrm{HCl} \mathrm{pH} 8.5,5 \mathrm{mM} \mathrm{MgCl}_{2}, 250 \mu \mathrm{M}$ ATP, $100 \mu \mathrm{M}$ UDPMurNAc dipeptide (UDP-MurNAc-L-ala-D-glu) and $1 \mathrm{mM} m$-DAP at $37^{\circ} \mathrm{C}$. The absorbance was measured at $635 \mathrm{~nm}$ using FLUOstar Optima plate reader (BMG Labtech). Wild type MurE was used as a positive control in all the experiments for comparison with the mutant proteins. The conditions were kept constant to help compare the results across different mutants and wild type protein. Absorbance values were corrected for background absorbance of reaction mixtures and any non-enzymatic hydrolysis of ATP in the absence of the enzyme. In addition, individual components of the assay were checked with the assay reagents to monitor the background absorbance. A standard phosphate curve was generated using the inorganic phosphate provided along with the kit to calculate the specific activity. All assays were performed in triplicate. The specific activity of the protein is expressed as picomoles of inorganic phosphate released during the reaction per minute per milligram of the recombinant protein.

\section{Protein dependence}

Protein dependence was determined for all the mutant proteins in addition to wild type. Protein was diluted in $25 \mathrm{mM}$ Bis-tris propane $\cdot \mathrm{HCl}(\mathrm{pH} 8.5)$ according to the quantity to be used. The amount of the protein used for the assay ranged 25-200 ng for wild type, D392A and MurEtr, and 100-1200 ng for rest of the mutants.

\section{Determination of the kinetic constants}

For determination of the kinetic constants, the assay was done at various concentrations of one substrate and fixed concentration of the others. For ATP, UMAG and $m$-DAP, varying concentrations of ATP at $10-500 \mu \mathrm{M}$, UMAG at 5-250 $\mu \mathrm{M}$ and $m$-DAP at 25-2500 $\mu \mathrm{M}$ with fixed concentrations of $250 \mu \mathrm{M}$ ATP, $100 \mu \mathrm{M}$ UMAG and $1000 \mu \mathrm{M} \mathrm{m}$ DAP respectively were used. The protein quantity used for all subsequent assays were $100 \mathrm{ng}$ of wild type, $700 \mathrm{ng}$ of N449D and $\mathrm{K} 157 \mathrm{~A}, 500 \mathrm{ng}$ of R451A, $150 \mathrm{ng}$ of E220A, $600 \mathrm{ng}$ of D392A and $100 \mathrm{ng}$ of MurEtr. Data was fitted to the Hanes' plot equation $[\mathrm{S}] / \mathrm{v}=$ $1 / V_{\max }^{*}[\mathrm{~S}]+K_{\mathrm{m}} / V_{\max }$ to calculate apparent $K_{m}$ and $V_{\max }$ by linear regression.

\section{Determination of substrate specificity}

Different amino acids, nucleotides and MurNAc substitutes were used to determine the substrate specificity of mutants of MurE. $1000 \mu \mathrm{M} \mathrm{m}$ DAP or other amino acids, $250 \mu \mathrm{M}$ ATP or other nucleotides, $100 \mu \mathrm{M}$ UDP-MurNAc-L-ala-D-glu or other MurNAc substitutes were added to the assay mix to initiate the reaction at $37^{\circ} \mathrm{C}$ for $30 \mathrm{~min}$. The rest of the assay set up was as described earlier. In all cases, the experiment was done in triplicate and the standard deviations are indicated by error bars $(n=3)$.

\section{Effect of ATP, $m$-DAP and UDP-MurNAc-L-ala-D-glu on ATP hydrolysis}

Complete and control reactions were set up as described previously. Three reactions were set up, first with ATP and enzyme, second with ATP, UDP-MurNAc-L-ala-D-glu and enzyme, third with ATP,
$m$-DAP and enzyme. The rest of the assay set up was as described earlier.

\section{Crystal structure of ternary complex}

Crystals of the MurE ternary complex were grown in the presence of $0.25 \mathrm{mM}$ ADP, $0.25 \mathrm{mM}$ UDP-MurNAc-L-ala-D-glu, $0.35 \mathrm{M} \mathrm{MgCl}_{2}$, $0.1 \mathrm{M}$ Tris $\mathrm{pH} 8.5$ and 13\% PEG 8000. Data was collected on beamline 104 at the Diamond Light Source. The structure was solved using the MurE/dipeptide binary complex 2 wtz (Basavannacharya et al., 2010) as a starting model for refinement and figures were prepared using CCP4MG.

\section{ACKNOWLEDGEMENTS}

This work was supported by grants from UK Medical Research Council (MRC) New Investigators Research Grant to Dr. Sanjib Bhakta (grant reference number G0801956). Chandrakala Basavannacharya acknowledges the ORS/BORS award from Birkbeck. Paul R. Moody is a Wellcome Trust Program Ph.D student. The funders have no active role in design, execution or preparation for publication of this work. We thank the UK Bacterial Cell Wall Biosynthesis Network (UK-BaCWAN) for supplying the uridine sugar precursors. The structure of the MurE/dipeptide/ADP complex has been submitted to the RCSB with code 2xja. We thank the beamline scientists at the Diamond Light Source for their support.

\section{ABBREVIATIONS}

FolC, folylpoly- $\gamma$-L-glutamate synthetase; Mpl, UDP-Nacetylmuramate:L-alanyl- $\gamma-D$-glutamyl-meso-diaminopimelate ligase; MurC, UDP-N-acetylmuramate:L-alanine ligase; MurD, UDP-N-acetylmuramoyl-L-alanine:D-glutamate ligase; MurE, UDP$\mathrm{N}$-acetylmuramoyl-L-alanyl-D-glutamate:meso-diaminopimelate ligase; MurF, UDP-N-acetylmuramoyl-L-alanyl-D-glutamyl-mesodiaminopimelate:D-alanyl-D-alanine ligase; UMAG, UDP-Nacetylmuramoyl-L-alanyl-D-glutamate; TDR-TB, totally drug-resistant tuberculosis

\section{REFERENCES}

Barreteau, H., Kovac, A., Boniface, A., Sova, M., Gobec, S., and Blanot, D. (2008). Cytoplasmic steps of peptidoglycan biosynthesis. FEMS Microbiol Rev 32, 168-207.

Basavannacharya, C., Robertson, G., Munshi, T., Keep, N.H., and Bhakta, S. (2010). ATP-dependent MurE ligase in Mycobacterium tuberculosis: biochemical and structural characterisation. Tuberculosis (Edinb) 90, 16-24.

Bertrand, J.A., Auger, G., Fanchon, E., Martin, L., Blanot, D., van Heijenoort, J., and Dideberg, O. (1997). Crystal structure of UDP$\mathrm{N}$-acetylmuramoyl-L-alanine:D-glutamate ligase from Escherichia coli. EMBO J 16, 3416-3425.

Bertrand, J.A., Auger, G., Martin, L., Fanchon, E., Blanot, D., Le Beller, D., van Heijenoort, J., and Dideberg, O. (1999). Determination of the MurD mechanism through crystallographic analysis of enzyme complexes. J Mol Biol 289, 579-590.

Bognar, A.L., Osborne, C., and Shane, B. (1987). Primary structure of the Escherichia coli folC gene and its folylpolyglutamate synthetase-dihydrofolate synthetase product and regulation of 
expression by an upstream gene. J Biol Chem 262, 12337-12343.

Boniface, A., Bouhss, A., Mengin-Lecreulx, D., and Blanot, D. (2006). The MurE synthetase from Thermotoga maritima is endowed with an unusual D-lysine adding activity. J Biol Chem 281, 15680-15686.

Bouhss, A., Dementin, S., Parquet, C., Mengin-Lecreulx, D., Bertrand, J.A., Le Beller, D., Dideberg, O., van Heijenoort, J., and Blanot, D. (1999). Role of the ortholog and paralog amino acid invariants in the active site of the UDP-MurNAc-L-alanine:Dglutamate ligase (MurD). Biochemistry 38, 12240-12247.

Bouhss, A., Mengin-Lecreulx, D., Blanot, D., van Heijenoort, J., and Parquet, C. (1997). Invariant amino acids in the Mur peptide synthetases of bacterial peptidoglycan synthesis and their modification by site-directed mutagenesis in the UDP-MurNAc:Lalanine ligase from Escherichia coli. Biochemistry 36, 11556-11563.

Bradford, M.M. (1976). A rapid and sensitive method for the quantitation of microgram quantities of protein utilizing the principle of protein-dye binding. Anal Biochem 72, 248-254.

Consaul, S.A., Wright, L.F., Mahapatra, S., Crick, D.C., and Pavelka, M.S. Jr. (2005). An unusual mutation results in the replacement of diaminopimelate with lanthionine in the peptidoglycan of a mutant strain of Mycobacterium smegmatis. J Bacteriol 187, 1612-1620.

Dementin, S., Bouhss, A., Auger, G., Parquet, C., Mengin-Lecreulx, D., Dideberg, O., van Heijenoort, J., and Blanot, D. (2001). Evidence of a functional requirement for a carbamoylated lysine residue in MurD, MurE and MurF synthetases as established by chemical rescue experiments. Eur J Biochem 268, 5800-5807.

Eveland, S.S., Pompliano, D.L., and Anderson, M.S. (1997). Conditionally lethal Escherichia coli murein mutants contain point defects that map to regions conserved among murein and folyl poly-gamma-glutamate ligases: identification of a ligase superfamily. Biochemistry 36, 6223-6229.

Glaser, P., Munier, H., Gilles, A.M., Krin, E., Porumb, T., Bârzu, O., Sarfati, R., Pellecuer, C., and Danchin, A. (1991). Functional consequences of single amino acid substitutions in calmodulinactivated adenylate cyclase of Bordetella pertussis. EMBO $\mathrm{J} 10$, 1683-1688.

Glauner, B., Höltje, J.V., and Schwarz, U. (1988). The composition of the murein of Escherichia coli. J Biol Chem 263, 10088-10095.

Gordon, E., Flouret, B., Chantalat, L., van Heijenoort, J., MenginLecreulx, D., and Dideberg, O. (2001). Crystal structure of UDP-Nacetylmuramoyl-L-alanyl-D-glutamate: meso-diaminopimelate ligase from Escherichia coli. J Biol Chem 276, 10999-11006.

Ikeda, M., Wachi, M., Jung, H.K., Ishino, F., and Matsuhashi, M. (1990). Nucleotide sequence involving murG and murC in the mra gene cluster region of Escherichia coli. Nucleic Acids Res 18,
4014.

Inoue, M., Hamada, S., Ooshima, T., Kotani, S., and Kato, K. (1979). Chemical composition of Streptococcus mutans cell walls and their susceptibility to Flavobacterium L-11 enzyme. Microbiol Immunol 23, 319-328.

Kawamoto, I., Oka, T., and Nara, T. (1981). Cell wall composition of Micromonospora olivoasterospora, Micromonospora sagamiensis, and related organisms. J Bacteriol 146, 527-534.

Laemmli, U.K. (1970). Cleavage of structural proteins during the assembly of the head of bacteriophage T4. Nature 227, 680-685.

Mahapatra, S., Scherman, H., Brennan, P.J., and Crick, D.C. (2005a). $\mathrm{N}$ Glycolylation of the nucleotide precursors of peptidoglycan biosynthesis of Mycobacterium spp. is altered by drug treatment. J Bacteriol 187, 2341-2347.

Mengin-Lecreulx, D., Blanot, D., and van Heijenoort, J. (1994). Replacement of diaminopimelic acid by cystathionine or lanthionine in the peptidoglycan of Escherichia coli. J Bacteriol 176, $4321-4327$.

Mitchell, C., and Oliver, D. (1993). Two distinct ATP-binding domains are needed to promote protein export by Escherichia coli SecA ATPase. Mol Microbiol 10, 483-497.

Nanninga, N. (1991). Cell division and peptidoglycan assembly in Escherichia coli. Mol Microbiol 5, 791-795.

Sambrook, J., and Russell, D.W. (2001). Molecular cloning: a laboratory manual, 3rd ed. edn (Cold Spring Harbor, N.Y., Cold Spring Harbor Laboratory Press).

Schleifer, K.H., and Kandler, O. (1972). Peptidoglycan types of bacterial cell walls and their taxonomic implications. Bacteriol Rev 36, 407-477.

Story, R.M., and Steitz, T.A. (1992). Structure of the recA protein-ADP complex. Nature 355, 374-376.

van der Wolk, J.P., Klose, M., de Wit, J.G., den Blaauwen, T., Freudl, R., and Driessen, A.J. (1995). Identification of the magnesiumbinding domain of the high-affinity ATP-binding site of the Bacillus subtilis and Escherichia coli SecA protein. J Biol Chem 270, 18975-18982.

Vasstrand, E., Jensen, H.B., and Miron, T. (1980). Microbore singlecolumn analysis of amino acids and amino sugars specific to bacterial cell wall peptidoglycans. Anal Biochem 105, 154-158.

Velayati, A.A., Masjedi, M.R., Farnia, P., Tabarsi, P., Ghanavi, J., Ziazarifi, A.H., and Hoffner, S.E. (2009). Emergence of new forms of totally drug-resistant tuberculosis bacilli: super extensively drugresistant tuberculosis or totally drug-resistant strains in iran. Chest 136, $420-425$.

Walker, J.E., Saraste, M., Runswick, M.J., and Gay, N.J. (1982). Distantly related sequences in the alpha- and beta-subunits of ATP synthase, myosin, kinases and other ATP-requiring enzymes and a common nucleotide binding fold. EMBO J 1, 945-951. 